\title{
Exponential Synchronization of Chaotic Xian System Using Linear Feedback Control
}

\author{
J. Humberto Pérez-Cruz $\mathbb{D},{ }^{1}$ Pedro A. Tamayo-Meza $\mathbb{D},{ }^{1}$ Maricela Figueroa $\mathbb{D},{ }^{1}$ \\ Ramón Silva-Ortigoza $\left(\mathbb{D},{ }^{2}\right.$ Mario Ponce-Silva, ${ }^{3}$ R. Rivera-Blas, ${ }^{2}$ and Mario Aldape-Pérez ${ }^{2}$ \\ ${ }^{1}$ Sección de Estudios de Posgrado e Investigación, Escuela Superior de Ingeniería Mecánica y Eléctrica, Unidad Azcapotzalco, \\ Instituto Politécnico Nacional, Ciudad de México 02250, Mexico \\ ${ }^{2}$ Área de Mecatrónica, Centro de Innovación y Desarrollo Tecnológico en Cómputo, Instituto Politécnico Nacional, \\ Ciudad de México 07700, Mexico \\ ${ }^{3}$ Departamento de Ingeniería Electrónica, Tecnológico Nacional de México, CENIDET, Cuernavaca 62490, Mexico
}

Correspondence should be addressed to J. Humberto Pérez-Cruz; jhperez@ipn.mx

Received 28 January 2019; Revised 3 June 2019; Accepted 23 June 2019; Published 25 July 2019

Academic Editor: Mohammed Chadli

Copyright (C) 2019 J. Humberto Pérez-Cruz et al. This is an open access article distributed under the Creative Commons Attribution License, which permits unrestricted use, distribution, and reproduction in any medium, provided the original work is properly cited.

\begin{abstract}
In this paper, a new linear feedback controller for synchronization of two identical chaotic systems in a master-slave configuration is presented. This controller requires knowing a priori Lipschitz constant of the nonlinear function of the chaotic system on its attractor. The controller development is based on an algebraic Riccati equation. If the gain matrix and the matrices of Riccati equation are selected in such a way that a unique positive definite solution is obtained for this equation, then, with respect to previous works, a stronger result can be guaranteed here: the exponential convergence to zero of the synchronization error. Additionally, the nonideal case is also studied, that is, when unmodeled dynamics and/or disturbances are present in both master system and slave system. On this new condition, the synchronization error does not converge to zero anymore. However, it is still possible to guarantee the exponential convergence to a bounded zone. Numerical simulation confirms the satisfactory performance of the suggested approach.
\end{abstract}

\section{Introduction}

The problem of the unidirectional synchronization of chaotic systems consists of finding an appropriate control law such that when this is applied to a system with coupled inputs called "slave" or "response," such system follows the dynamics of an autonomous chaotic system called "master" or "drive" [1-11]. This proper control action is necessary because, without it, two identical autonomous chaotic systems could never be synchronized due to their high sensitivity to initial conditions [12-18].

During the last three decades, several strategies have been proposed to solve the synchronization problem when the structure and the parameters of both chaotic systems are known. One of these approaches is active control [1931]. In this approach, the controller is selected based on the synchronization error dynamics in such a way that the nonlinearities are compensated and the dynamics equations are decoupled [32]. Another approach is nonlinear control [3340]. In this technique, given a Lyapunov function candidate $V$, the control law is selected considering that the first derivative of $V$ must be compelled to be negative definite [41]. Hence, the asymptotic convergence to zero of the synchronization error can be guaranteed. The aforementioned strategies present two drawbacks: (a) their practical implementation can be difficult, particularly for analog systems; (b) the controller can be expensive with respect to the consumption of energy. These problems can be avoided if a linear feedback controller is used [42-45]. This kind of controller is formed by the product of a gain matrix $\mathbf{K}$ and the negative synchronization error, $-\mathbf{e}$, that is, $\mathbf{u}=-\mathbf{K e}$. In [46], Wang et al. proposed an approach for synchronization of Chen system using linear feedback control. Given a proper Lyapunov function candidate, they could express the first time derivative of this function as a quadratic form of the synchronization error absolute value. Thus, by taking 
into account the conditions on which this quadratic form is negative definite, they could guarantee the asymptotic convergence to zero. This result was obtained by using only a unique control input. Based on this technique, the synchronization of the unified chaotic system and Lü system was presented in [47] and [48], respectively. It is important to mention that both Chen system and unified system are formed by five linear terms and two cross-product terms. Lü system is formed by four linear terms and two crossproduct terms. Both cross-product terms are exactly the same expression in the three cases. By using Wang's technique, Yassen synchronized a four-scroll chaotic system formed by three linear terms and three cross-product terms [49]. However, to be able to guarantee the asymptotic convergence, Yassen had to use three control inputs. Likewise, Liu chaotic system was synchronized in [50] with three control inputs and using the same procedure but applied from a more general perspective. Based on the same technique but with a unique control input, hyperchaotic Lorenz-Stenflo system formed by eight linear terms and two cross-product terms, and Lü hyperchaotic system formed by seven linear terms and two cross-product terms were synchronized in [51] and [52], respectively. In spite of the aforementioned successful applications, this technique has two main disadvantages: (a) the design procedure must be particularized to each system; (b) the technique is valid only for a very constrained class of systems. On the other hand, by using linearization and Lyapunov's direct methods and linear feedback control, a sufficient synchronization criterion was presented in [42] for the case of generalized Lorenz systems. A wider class (but still restrictive) of systems were considered in [44] for synchronization based on linear feedback control. In all the aforementioned works, only the asymptotic convergence of the synchronization error can be guaranteed. To overcome this situation and the limitations of Wang's technique, in this paper, a new linear feedback controller based on a matrix Riccati equation is presented. This procedure requires knowing a priori Lipschitz constant of nonlinear function of the chaotic system on its attractor. If the gain matrix $\mathbf{K}$ and the matrices of the Riccati equation are selected in such a way that this equation has a unique positive definite solution, then a stronger result can here be established: the exponential convergence to zero of the synchronization error. The proposed strategy can be applied to a very wide class of systems. However, for simplicity, the attention is focused on a system recently reported by Xian et al in [53]. Despite the similarity between this system and the system synchronized by Yassen in [49] (the main difference is only a unique crossproduct term), Wang's technique is not able to handle this new system.

Throughout this paper, given a vector $\mathbf{w} \in \mathfrak{R}^{n},\|\mathbf{w}\|$ denotes the Euclidean norm of $\mathbf{w}$, that is, $\|\mathbf{w}\|=\sqrt{\mathbf{w}^{T} \mathbf{w}}$; given a matrix $\mathbf{C} \in \Re^{n \times n}, \lambda_{\min }(\mathbf{C})$ and $\lambda_{\text {max }}(\mathbf{C})$ denote the minimum and maximum eigenvalues of $\mathbf{C}$, respectively; $\|\mathbf{C}\|$ denotes the two-norm of $\mathbf{C}$, that is, $\|\mathbf{C}\|=\sqrt{\lambda_{\max }\left(\mathbf{C}^{T} \mathbf{C}\right)}$.

\section{System Description}

The system proposed by Xian [53] is a new generalized third-order Lü chaotic system formed by three linear terms and four cross-product terms. The system can be described as

$$
\begin{aligned}
& \dot{x}=-a x+x z+y z \\
& \dot{y}=b y-x z \\
& \dot{z}=-c z+x y
\end{aligned}
$$

where $x, y$, and $z$ are the system states and $a, b$, and $c$ are constant parameters. This system shows chaotic behavior for the values $a=5, b=7, c=3$ and the initial condition $x(0)=$ $-1, y(0)=-1$, and $z(0)=-2$. In Figure 1 , the corresponding attractor is shown. The time series for the states $x, y$, and $z$ during the first twenty seconds of numerical simulation are plotted in Figure 2.

It can be noted that, by using vector notation, system (1) can succinctly be represented as follows:

$$
\dot{\mathbf{w}}=\mathbf{A w}+\mathbf{f}(\mathbf{w})
$$

where

$$
\begin{aligned}
\mathbf{w} & :=\left[\begin{array}{lll}
x & y & z
\end{array}\right]^{T} \\
\mathbf{A} & :=\left[\begin{array}{ccc}
-a & 0 & 0 \\
0 & b & 0 \\
0 & 0 & -c
\end{array}\right] \\
\mathbf{f}(\mathbf{w}):= & {\left[\begin{array}{c}
x z+y z \\
-x z \\
x y
\end{array}\right] }
\end{aligned}
$$

\section{Problem Formulation}

The simpler structure for synchronization of chaotic systems is master-slave configuration. In this one, a slave chaotic system with control inputs must follow the dynamic behavior of an autonomous master chaotic system. For system (2), the corresponding master system can be represented simply as

$$
\dot{\mathbf{w}}_{m}=\mathbf{A} \mathbf{w}_{m}+\mathbf{f}\left(\mathbf{w}_{m}\right)
$$

where $\mathbf{w}_{m}:=\left[\begin{array}{lll}x_{m} & y_{m} & z_{m}\end{array}\right]^{T}$ and subscript $m$ denotes "master." The corresponding slave system for system (2) is given by

$$
\dot{\mathbf{w}}_{s}=\mathbf{A} \mathbf{w}_{s}+\mathbf{f}\left(\mathbf{w}_{s}\right)+\mathbf{u}
$$

where $\mathbf{w}_{s}:=\left[\begin{array}{lll}x_{s} & y_{s} & z_{s}\end{array}\right]^{T}, \mathbf{u}:=\left[\begin{array}{lll}u_{1} & u_{2} & u_{3}\end{array}\right]^{T}, u_{1}, u_{2}$, and $u_{3}$ are control inputs, and the subscript $s$ denotes "slave." Define the synchronization error [54] as

$$
\mathbf{e}:=\mathbf{w}_{s}-\mathbf{w}_{m}
$$

Thus, the problem of synchronization based on linear feedback control for systems (7) and (6) consists of finding an appropriate control law of the form

$$
\mathbf{u}=-\mathbf{K e}
$$




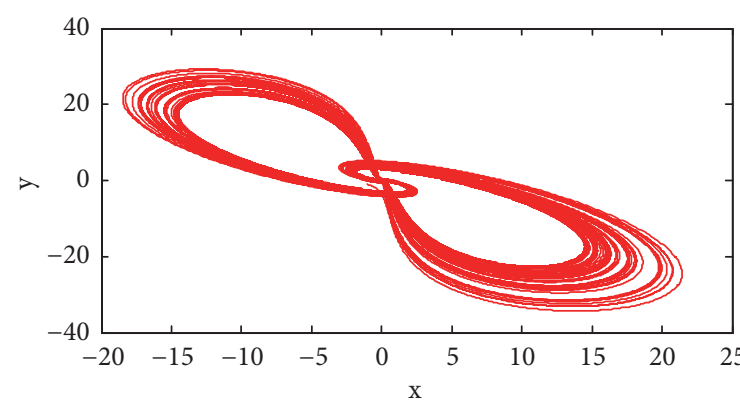

(a)

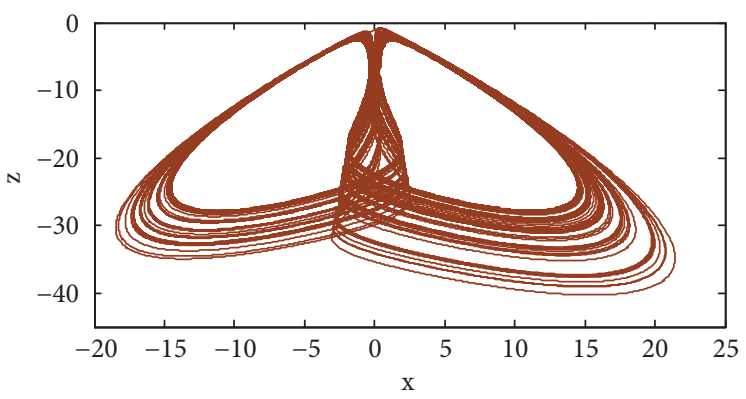

(c)

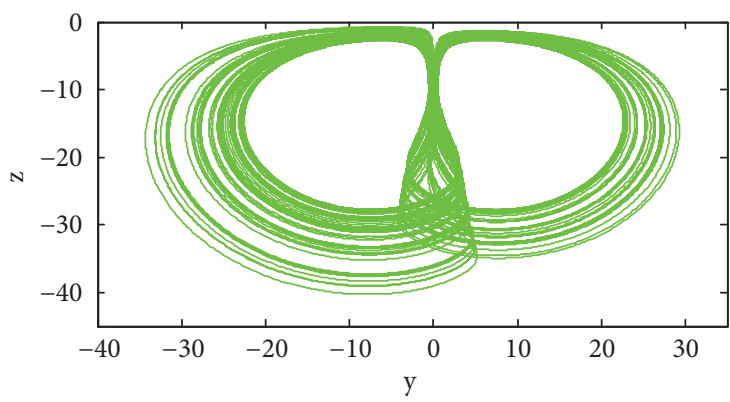

(b)

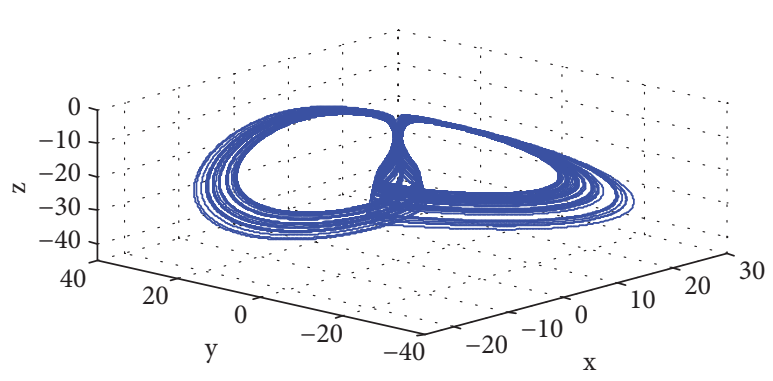

(d)

Figure 1: Attractor for Xian system: (a) $x-y$ phase plane, (b) $y-z$ phase plane, (c) $x-z$ phase plane, and (d) phase space.

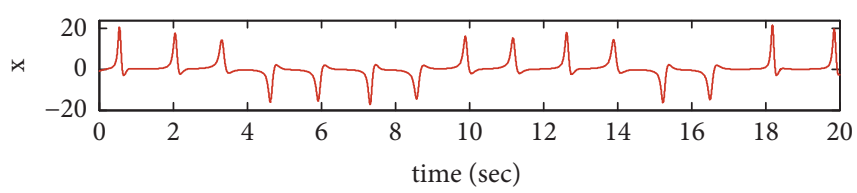

(a)

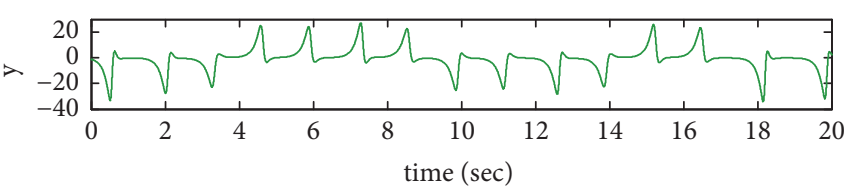

(b)

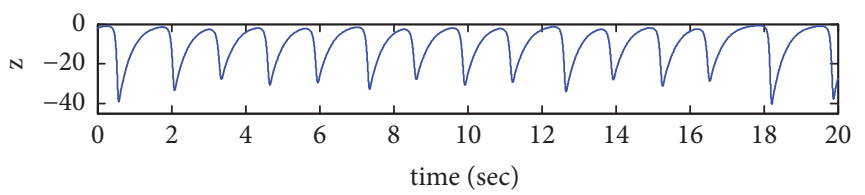

(c)

Figure 2: Time evolution of states of Xian system for first 20 seconds: (a) state $x$, (b) state $y$, and (c) state $z$.

where

$$
\mathbf{K}=\left[\begin{array}{ccc}
k_{1} & 0 & 0 \\
0 & k_{2} & 0 \\
0 & 0 & k_{3}
\end{array}\right]
$$

and $k_{1}, k_{2}$, and $k_{3}$ are real constants selectable by the designer in such a way that $\lim _{t \rightarrow \infty} \mathbf{e}=\mathbf{0}$.

\section{Background Results}

In this section, some basic definitions and results are reviewed briefly.

Definition 1 (see [55-57]). A function $\mathbf{f}(\mathbf{w}): \mathfrak{R}^{n} \longrightarrow \mathfrak{R}^{n}$ is said to be locally Lipschitz on $D \subset \mathfrak{R}^{n}$ if there exists a constant $\gamma$ (known as Lipschitz constant) such that, for all $\mathbf{w}_{1}, \mathbf{w}_{2} \in D$, the following inequality holds:

$$
\left\|\mathbf{f}\left(\mathbf{w}_{1}\right)-\mathbf{f}\left(\mathbf{w}_{2}\right)\right\| \leq \gamma\left\|\mathbf{w}_{1}-\mathbf{w}_{2}\right\|
$$

Finally, $\mathbf{f}$ is said to be globally Lipschitz if it satisfies (11) with $D=\mathfrak{R}^{n}$.

Lemma 2 (see $[56,57]$ ). If a function $\mathbf{f}: \mathfrak{R}^{n} \longrightarrow \mathfrak{R}^{n}$ is continuously differentiable on a set $D \subset \mathfrak{R}^{n}$, then it is locally Lipschitz on D.

Based on Lemma 2, a procedure can be established in order to calculate the Lipschitz constant $\gamma$ [56]. For example, consider the function

$$
\mathbf{g}(\mathbf{w})=\left[\begin{array}{c}
w_{1} w_{2}^{2}-w_{2} \\
w_{1} w_{2}
\end{array}\right]
$$


on the set $D=\left\{\left(w_{1}, w_{2}\right) \mid-4 \leq w_{1} \leq 2,-1 \leq w_{2} \leq 3\right\}$. Let us define $w_{1, \max }$ and $w_{2, \text { max }}$ such that $\left|w_{1}\right| \leq w_{1, \max }$ and $\left|w_{2}\right| \leq w_{2, \max }$. Then, on $D, w_{1, \max }=4$ and $w_{2, \max }=3$. On the other hand, the Jacobian matrix of $\mathbf{g}$ is given by

$$
\frac{\partial \mathbf{g}}{\partial \mathbf{w}}=\left[\begin{array}{cc}
w_{2}^{2} & 2 w_{1} w_{2}-1 \\
w_{2} & w_{1}
\end{array}\right]
$$

Let us define the matrix $\mathbf{G}$ as

$$
\mathbf{G}:=\left.\frac{\partial \mathbf{g}}{\partial \mathbf{w}}\right|_{\max }
$$

That is, $\mathbf{G}$ is formed by the maximum absolute values of each corresponding component in (13). Consequently,

$$
\mathbf{G}=\left[\begin{array}{cc}
9 & 25 \\
3 & 4
\end{array}\right]
$$

Finally, a Lipschitz constant for $\mathbf{g}$ (12) on the set $D$ can be taken as

$$
\gamma=\|\mathbf{G}\|=26.9984
$$

Lemma 3 (see [58]). For any vectors $\mathbf{w}_{1}, \mathbf{w}_{2} \in \Re^{n}$ and any positive definite matrix $\boldsymbol{\Lambda}^{T}=\boldsymbol{\Lambda}>0, \boldsymbol{\Lambda} \in \mathfrak{R}^{n \times n}$, the following inequality holds:

$$
\mathbf{w}_{1}^{T} \mathbf{w}_{2}+\mathbf{w}_{2}^{T} \mathbf{w}_{1} \leq \mathbf{w}_{1}^{T} \boldsymbol{\Lambda} \mathbf{w}_{1}+\mathbf{w}_{2}^{T} \Lambda^{-1} \mathbf{w}_{2}
$$

Lemma 4 (see [58]). The matrix Riccati equation

$$
\mathbf{A}_{0}^{T} \mathbf{P}+\mathbf{P A}_{0}+\mathbf{P R P}+\mathbf{Q}=\mathbf{0}
$$

with known constant matrices $\mathbf{A}_{0}, \mathbf{R}, \mathbf{Q} \in \mathfrak{R}^{n \times n}$ has a unique positive definite solution $\mathbf{P}^{T}=\mathbf{P}>0, \mathbf{P} \in \mathfrak{R}^{n \times n}$ if the following conditions are satisfied:

(a) $\mathbf{R}^{T}=\mathbf{R}>0, \mathbf{Q}^{T}=\mathbf{Q}>0$

(b) $\mathbf{A}_{\mathbf{0}}$ is Hurwitz

(c) The pair $\left(\mathbf{A}_{0}, \mathbf{R}^{1 / 2}\right)$ is controllable

(d) The pair $\left(\mathbf{Q}^{1 / 2}, \mathbf{A}_{0}\right)$ is observable

(e) The following matrix inequality holds:

$$
\begin{aligned}
& \frac{1}{4}\left(\mathbf{A}_{0}^{T} \mathbf{R}^{-1}-\mathbf{R}^{-1} \mathbf{A}_{0}\right) \mathbf{R}\left(\mathbf{A}_{0}^{T} \mathbf{R}^{-1}-\mathbf{R}^{-1} \mathbf{A}_{0}\right)^{T} \\
& \quad \leq \mathbf{A}_{0}^{T} \mathbf{R}^{-1} \mathbf{A}_{0}-\mathbf{Q}
\end{aligned}
$$

\section{Main Results}

In this section, the conditions under which the control law (9) can synchronize system (7) with respect to system (6) are found. First, the dynamics of the synchronization error is determined. By taking the first derivative of (8), we obtain

$$
\dot{\mathbf{e}}=\dot{\mathbf{w}}_{s}-\dot{\mathbf{w}}_{m}
$$

By substituting (7) and (6) into (20) and taking into account that the control law has the form given in (9), we get

$$
\dot{\mathbf{e}}=(\mathbf{A}-\mathbf{K}) \mathbf{e}+\mathbf{f}\left(\mathbf{w}_{s}\right)-\mathbf{f}\left(\mathbf{w}_{m}\right)
$$

Let us define

$$
\mathbf{A}_{0}:=\mathbf{A}-\mathbf{K}
$$

Thus, (21) becomes

$$
\dot{\mathbf{e}}=\mathbf{A}_{0} \mathbf{e}+\mathbf{f}\left(\mathbf{w}_{s}\right)-\mathbf{f}\left(\mathbf{w}_{m}\right)
$$

To evaluate the stability of the synchronization error dynamics (23), the following Lyapunov function candidate is proposed:

$$
V=\mathbf{e}^{T} \mathbf{P e}
$$

where $\mathbf{P}$ is a positive definite matrix to be found. The first time derivative of (24) is calculated as

$$
\dot{V}=\dot{\mathbf{e}}^{T} \mathbf{P e}+\mathbf{e}^{T} \mathbf{P e}
$$

By substituting (23) into (25) and after some operations, we get

$$
\begin{aligned}
\dot{V}= & \mathbf{e}^{T} \mathbf{A}_{0}^{T} \mathbf{P e}+\mathbf{e}^{T} \mathbf{P} \mathbf{A}_{0} \mathbf{e}+\left(\mathbf{f}\left(\mathbf{w}_{s}\right)-\mathbf{f}\left(\mathbf{w}_{m}\right)\right)^{T} \mathbf{P e} \\
& +\mathbf{e}^{T} \mathbf{P}\left(\mathbf{f}\left(\mathbf{w}_{s}\right)-\mathbf{f}\left(\mathbf{w}_{m}\right)\right)
\end{aligned}
$$

Now, let us consider the last two terms of (26):

$$
\left(\mathbf{f}\left(\mathbf{w}_{s}\right)-\mathbf{f}\left(\mathbf{w}_{m}\right)\right)^{T} \mathbf{P e}+\mathbf{e}^{T} \mathbf{P}\left(\mathbf{f}\left(\mathbf{w}_{s}\right)-\mathbf{f}\left(\mathbf{w}_{m}\right)\right)
$$

By using Lemma 3, (27) can be bounded as

$$
\begin{aligned}
& \left(\mathbf{f}\left(\mathbf{w}_{s}\right)-\mathbf{f}\left(\mathbf{w}_{m}\right)\right)^{T} \mathbf{P e}+\mathbf{e}^{T} \mathbf{P}\left(\mathbf{f}\left(\mathbf{w}_{s}\right)-\mathbf{f}\left(\mathbf{w}_{m}\right)\right) \\
& \quad \leq \mathbf{e}^{T} \mathbf{P} \boldsymbol{\Lambda} \mathbf{P e} \\
& \quad+\left(\mathbf{f}\left(\mathbf{w}_{s}\right)-\mathbf{f}\left(\mathbf{w}_{m}\right)\right)^{T} \boldsymbol{\Lambda}^{-1}\left(\mathbf{f}\left(\mathbf{w}_{s}\right)-\mathbf{f}\left(\mathbf{w}_{m}\right)\right)
\end{aligned}
$$

where $\Lambda$ is a definite positive matrix selectable by the designer. Besides, considering that $\mathbf{f}$ is locally Lipschitz on the attractor of the autonomous system (6), with Lipschitz constant $\gamma$, then the following can be established:

$$
\left\|\mathbf{f}\left(\mathbf{w}_{s}\right)-\mathbf{f}\left(\mathbf{w}_{m}\right)\right\| \leq \gamma\left\|\mathbf{w}_{s}-\mathbf{w}_{m}\right\|
$$

and

$$
\left(\mathbf{f}\left(\mathbf{w}_{s}\right)-\mathbf{f}\left(\mathbf{w}_{m}\right)\right)^{T} \boldsymbol{\Lambda}^{-1}\left(\mathbf{f}\left(\mathbf{w}_{s}\right)-\mathbf{f}\left(\mathbf{w}_{m}\right)\right) \leq \gamma^{2} \mathbf{e}^{T} \boldsymbol{\Lambda}^{-1} \mathbf{e}
$$

By substituting (30) into (28) and the corresponding result into (26), the first time derivative of $V$ can be bounded by

$$
\dot{V} \leq \mathbf{e}^{T} \mathbf{A}_{0}^{T} \mathbf{P e}+\mathbf{e}^{T} \mathbf{P} \mathbf{A}_{0} \mathbf{e}+\mathbf{e}^{T} \mathbf{P} \boldsymbol{\Lambda} \mathbf{P e}+\gamma^{2} \mathbf{e}^{T} \boldsymbol{\Lambda}^{-1} \mathbf{e}
$$

By adding and subtracting the term $\mathbf{e}^{T} \mathbf{Q}_{0} \mathbf{e}$, where $\mathbf{Q}_{0}$ is a positive definite matrix, into the right hand side of (31), we obtain

$$
\begin{aligned}
\dot{V} & \leq\left(\mathbf{e}^{T} \mathbf{A}_{0}^{T} \mathbf{P e}+\mathbf{e}^{T} \mathbf{P} \mathbf{A}_{0} \mathbf{e}+\mathbf{e}^{T} \mathbf{P} \boldsymbol{\Lambda} \mathbf{P e}+\gamma^{2} \mathbf{e}^{T} \boldsymbol{\Lambda}^{-1} \mathbf{e}\right. \\
& \left.+\mathbf{e}^{T} \mathbf{Q}_{0} \mathbf{e}\right)-\mathbf{e}^{T} \mathbf{Q}_{0} \mathbf{e}
\end{aligned}
$$


Now, if we define

$$
\begin{aligned}
& \mathbf{R}:=\boldsymbol{\Lambda}, \\
& \mathbf{Q}:=\gamma^{2} \boldsymbol{\Lambda}^{-1}+\mathbf{Q}_{0},
\end{aligned}
$$

and the following matrix Riccati equation is formed:

$$
\mathbf{A}_{0}^{T} \mathbf{P}+\mathbf{P A}_{0}+\mathbf{P R} \mathbf{P}+\mathbf{Q}=\mathbf{0}
$$

and if the constant matrices $\mathbf{A}_{0}, \boldsymbol{\Lambda}, \mathbf{Q}_{0}$ are selected in such a way that the conditions of the Lemma 4 are satisfied, then (34) has a unique positive definite solution $\mathbf{P}$ and (32) becomes simply

$$
\dot{V} \leq-\mathbf{e}^{T} \mathbf{Q}_{0} \mathbf{e}
$$

From (35) and from second Lyapunov method, the asymptotic convergence to zero of e can be concluded. However, a stronger result can still be obtained. Let us consider that

$$
-\mathbf{e}^{T} \mathbf{Q}_{0} \mathbf{e}=-\mathbf{e}^{T} \mathbf{P}^{1 / 2} \mathbf{P}^{-1 / 2} \mathbf{Q}_{0} \mathbf{P}^{-1 / 2} \mathbf{P}^{1 / 2} \mathbf{e}
$$

By using Rayleigh inequality into (36), we can claim that

$$
-\mathbf{e}^{T} \mathbf{Q}_{0} \mathbf{e} \leq-\lambda_{\min }\left(\mathbf{P}^{-1 / 2} \mathbf{Q}_{0} \mathbf{P}^{-1 / 2}\right) \mathbf{e}^{T} \mathbf{P e}
$$

From the last inequality and by taking into account (35),

$$
\dot{V} \leq-\lambda_{\min }\left(\mathbf{P}^{-1 / 2} \mathbf{Q}_{0} \mathbf{P}^{-1 / 2}\right) \mathbf{e}^{T} \mathbf{P e}
$$

Let us define

$$
\zeta:=\lambda_{\min }\left(\mathbf{P}^{-1 / 2} \mathbf{Q}_{0} \mathbf{P}^{-1 / 2}\right)
$$

And from (24), (38) becomes

$$
\dot{V} \leq-\zeta V
$$

This implies that

$$
V \leq V(0) \exp (-\zeta t)
$$

By using twice Rayleigh inequality into (24), (41) becomes

$$
\lambda_{\min }(P) \mathbf{e}^{T} \mathbf{e} \leq \lambda_{\max }(P) \mathbf{e}^{T}(0) \mathbf{e}(0) \exp (-\zeta t)
$$

or

$$
\|\mathbf{e}\|^{2} \leq \frac{\lambda_{\max }(P)}{\lambda_{\min }(P)}\|\mathbf{e}(0)\|^{2} \exp (-\zeta t)
$$

By taking square root of both sides of inequality (43), we get

$$
\|\mathbf{e}\| \leq \sqrt{\frac{\lambda_{\max }(P)}{\lambda_{\min }(P)}}\|\mathbf{e}(0)\| \exp \left(-\frac{\zeta}{2} t\right)
$$

Finally, based on (44), we can conclude the exponential convergence to zero of the synchronization error e. Hence, the following theorem has been proven.
Theorem 5. If the function $\mathbf{f}$ in equations (6) and (7) is locally Lipschitz on the attractor of the autonomous system (6) with Lipschitz constant $\gamma$ and the gain $\mathbf{K}$ and the matrices $\mathbf{\Lambda}, \mathbf{Q}_{0}, \mathbf{A}_{0}=\mathbf{A}-\mathbf{K}, \mathbf{R}=\mathbf{\Lambda}, \mathbf{Q}=\gamma^{2} \boldsymbol{\Lambda}^{-1}+\mathbf{Q}_{0}$ are selected according to Lemma 4 in such a way that the matrix Riccati equation $\mathbf{A}_{0}^{T} \mathbf{P}+\mathbf{P A}_{0}+\mathbf{P R P}+\mathbf{Q}=\mathbf{0}$ has a unique positive definite solution $\mathbf{P}$ and the control law $\mathbf{u}=-\mathbf{K e}$ is applied to the slave system (7), then the synchronization error $\mathbf{e}=\mathbf{w}_{s}-\mathbf{w}_{m}$ converges exponentially to zero.

Now, a more realistic case can be considered when unmodeled dynamics and/or disturbances are present in both master system and slave system, that is,

$$
\begin{aligned}
& \dot{\mathbf{w}}_{m}=\mathbf{A} \mathbf{w}_{m}+\mathbf{f}\left(\mathbf{w}_{m}\right)+\psi_{\mathbf{m}} \\
& \dot{\mathbf{w}}_{s}=\mathbf{A} \mathbf{w}_{s}+\mathbf{f}\left(\mathbf{w}_{s}\right)+\mathbf{u}+\psi_{\mathbf{s}}
\end{aligned}
$$

Assumption 6. The terms $\psi_{\mathrm{m}}$ and $\psi_{\mathrm{s}}$ represent unknown unmodeled dynamics and/or disturbances. Although these terms are unknown, they must be bounded. Besides, it is not necessary to know the specific value for each bound.

Consider a function $\psi$ defined as

$$
\psi:=\psi_{\mathrm{s}}-\psi_{\mathrm{m}}
$$

which satisfies

$$
\boldsymbol{\psi}^{T} \boldsymbol{\Omega} \boldsymbol{\Psi} \bar{\Psi}
$$

where $\Omega$ is a definite positive matrix selectable by the designer and $\bar{\Psi}$ is a positive constant not necessarily a priori known.

Theorem 7. If the function $\mathbf{f}$ in equations (45) and (46) is locally Lipschitz on the attractor of the autonomous system (45) with Lipschitz constant $\gamma$ and the gain $\mathbf{K}$ and the matrices $\boldsymbol{\Lambda}, \mathbf{\Omega}, \mathbf{Q}_{0}, \mathbf{A}_{0}=\mathbf{A}-\mathbf{K}, \overline{\mathbf{R}}=\boldsymbol{\Lambda}+\mathbf{\Omega}^{-1}, \mathbf{Q}=\gamma^{2} \boldsymbol{\Lambda}^{-1}+\mathbf{Q}_{0}$ are selected according to Lemma 4 in such a way that the matrix Riccati equation $\mathbf{A}_{0}^{T} \mathbf{P}_{1}+\mathbf{P}_{1} \mathbf{A}_{0}+\mathbf{P}_{1} \overline{\mathbf{R}} \mathbf{P}_{1}+\mathbf{Q}=\mathbf{0}$ has a unique positive definite solution $\mathbf{P}_{1}$ and the control law $\mathbf{u}=-\mathbf{K e}$ is applied to the slave system (46), then the norm of the synchronization error $\|\mathbf{e}\|=\left\|\mathbf{w}_{s}-\mathbf{w}_{m}\right\|$ converges exponentially to a zone bounded by $\sqrt{\bar{\Psi} /\left(\alpha \lambda_{\min }\left(P_{1}\right)\right)}$ where $\alpha=\lambda_{\min }\left(\mathbf{P}_{1}^{-1 / 2} \mathbf{Q}_{0} \mathbf{P}_{1}^{-1 / 2}\right)$.

Proof. As the proof of this theorem is very similar to the proof of Theorem 5, only the main points will be presented. Given $\mathbf{e}=\mathbf{w}_{s}-\mathbf{w}_{m}$, the synchronization error dynamics is given by

$$
\dot{\mathbf{e}}=\mathbf{A}_{0} \mathbf{e}+\mathbf{f}\left(\mathbf{w}_{s}\right)-\mathbf{f}\left(\mathbf{w}_{m}\right)+\psi
$$

To analyze this dynamics, the following Lyapunov function candidate is proposed:

$$
V=\mathbf{e}^{T} \mathbf{P}_{1} \mathbf{e}
$$

The first time derivative of (50) can be expressed as

$$
\begin{aligned}
\dot{V} \leq & \mathbf{e}^{T} \mathbf{A}_{0}^{T} \mathbf{P}_{1} \mathbf{e}+\mathbf{e}^{T} \mathbf{P}_{1} \mathbf{A}_{0} \mathbf{e}+\mathbf{e}^{T} \mathbf{P}_{1} \Lambda \mathbf{P}_{1} \mathbf{e}+\gamma^{2} \mathbf{e}^{T} \boldsymbol{\Lambda}^{-1} \mathbf{e} \\
& +\psi^{T} \mathbf{P}_{1} \mathbf{e}+\mathbf{e}^{T} \mathbf{P}_{1} \psi
\end{aligned}
$$


By using Lemma 3 into $\psi^{T} \mathbf{P}_{1} \mathbf{e}+\mathbf{e}^{T} \mathbf{P}_{1} \boldsymbol{\psi}$ and inequality (48), it can be obtained that

$$
\psi^{T} \mathbf{P}_{1} \mathbf{e}+\mathbf{e}^{T} \mathbf{P}_{1} \psi \leq \mathbf{e}^{T} \mathbf{P}_{1} \boldsymbol{\Omega}^{-1} \mathbf{P}_{1} \mathbf{e}+\bar{\Psi}
$$

By substituting (52) into (51), we get

$$
\begin{aligned}
\dot{V} \leq & \mathbf{e}^{T} \mathbf{A}_{0}^{T} \mathbf{P}_{1} \mathbf{e}+\mathbf{e}^{T} \mathbf{P}_{1} \mathbf{A}_{0} \mathbf{e}+\mathbf{e}^{T} \mathbf{P}_{1} \boldsymbol{\Lambda} \mathbf{P}_{1} \mathbf{e}+\mathbf{e}^{T} \mathbf{P}_{1} \boldsymbol{\Omega}^{-1} \mathbf{P}_{1} \mathbf{e} \\
& +\gamma^{2} \mathbf{e}^{T} \boldsymbol{\Lambda}^{-1} \mathbf{e}+\bar{\Psi}
\end{aligned}
$$

By adding and subtracting the term $\mathbf{e}^{T} \mathbf{Q}_{0} \mathbf{e}$, where $\mathbf{Q}_{0}$ is a positive definite matrix, into the right hand side of (53), we obtain

$$
\begin{aligned}
\dot{V} & \leq\left(\mathbf{e}^{T} \mathbf{A}_{0}^{T} \mathbf{P}_{1} \mathbf{e}+\mathbf{e}^{T} \mathbf{P}_{1} \mathbf{A}_{0} \mathbf{e}+\mathbf{e}^{T} \mathbf{P}_{1} \boldsymbol{\Lambda} \mathbf{P}_{1} \mathbf{e}\right. \\
& \left.+\mathbf{e}^{T} \mathbf{P}_{1} \mathbf{\Omega}^{-1} \mathbf{P}_{1} \mathbf{e}+\gamma^{2} \mathbf{e}^{T} \Lambda^{-1} \mathbf{e}+\mathbf{e}^{T} \mathbf{Q}_{0} \mathbf{e}\right)-\mathbf{e}^{T} \mathbf{Q}_{0} \mathbf{e} \\
& +\bar{\Psi}
\end{aligned}
$$

By defining

$$
\begin{aligned}
& \overline{\mathbf{R}}:=\boldsymbol{\Lambda}+\mathbf{\Omega}^{-1}, \\
& \mathbf{Q}:=\gamma^{2} \boldsymbol{\Lambda}^{-1}+\mathbf{Q}_{0},
\end{aligned}
$$

the following matrix Riccati equation is formed:

$$
\mathbf{A}_{0}^{T} \mathbf{P}_{1}+\mathbf{P}_{1} \mathbf{A}_{0}+\mathbf{P}_{1} \overline{\mathbf{R}} \mathbf{P}_{1}+\mathbf{Q}=\mathbf{0}
$$

and if the constant matrices $\mathbf{A}_{0}, \boldsymbol{\Lambda}, \boldsymbol{\Omega}, \mathbf{Q}_{0}$ are selected using Lemma 4, then (56) has a unique positive definite solution $\mathbf{P}_{1}$ and (54) becomes simply

$$
\dot{V} \leq-\mathbf{e}^{T} \mathbf{Q}_{0} \mathbf{e}+\bar{\Psi}
$$

Now, it can be shown that

$$
-\mathbf{e}^{T} \mathbf{Q}_{0} \mathbf{e} \leq-\alpha \mathbf{e}^{T} \mathbf{P}_{1} \mathbf{e}
$$

where $\alpha=\lambda_{\min }\left(\mathbf{P}_{1}^{-1 / 2} \mathbf{Q}_{0} \mathbf{P}_{1}^{-1 / 2}\right)$. By substituting (58) into (57) and (50) into the resulting expression, we get

$$
\dot{V} \leq-\alpha V+\bar{\Psi}
$$

According to [59, 60], (59) implies that

$$
V \leq V(0) \exp (-\alpha t)+\frac{\bar{\Psi}}{\alpha}(1-\exp (-\alpha t))
$$

This means that

$$
\begin{aligned}
& \|\mathbf{e}\| \\
& \leq \sqrt{\frac{V(0)}{\lambda_{\text {min }}\left(P_{1}\right)} \exp (-\alpha t)+\frac{\bar{\Psi}}{\alpha \lambda_{\min }\left(P_{1}\right)}(1-\exp (-\alpha t))}
\end{aligned}
$$

The boundedness of e can be concluded from (61). Finally, by taking the limit on both sides of (61) as time tends to infinity, we can conclude the exponential convergence of $\|\mathbf{e}\|$ to a zone bounded by $\sqrt{\bar{\Psi} /\left(\alpha \lambda_{\min }\left(P_{1}\right)\right)}$.

\section{Numerical Simulation}

In order to use the result of Theorem 5, first, by numerical simulation, the maximum absolute value of each state of the autonomous system (6) is estimated as $x_{\max }=40, y_{\max }=$ 66.54, and $z_{\max }=74.1$. Next, the Lipschitz constant of function $\mathbf{f}$ on the attractor of the autonomous system (6) is determined. The Jacobian matrix of $\mathbf{f}$ is given by

$$
\frac{\partial \mathbf{f}}{\partial \mathbf{w}}=\left[\begin{array}{ccc}
z & z & x+y \\
-z & 0 & -x \\
y & x & 0
\end{array}\right]
$$

The matrix $\mathbf{F}$ formed by the maximum absolute values of each element of the corresponding Jacobian matrix on the attractor of the autonomous system (6) is calculated as

$$
\mathbf{F}=\left[\begin{array}{ccc}
z_{\max } & z_{\max } & x_{\max }+y_{\max } \\
z_{\max } & 0 & x_{\max } \\
y_{\max } & x_{\max } & 0
\end{array}\right]
$$

The two-norm of $\mathbf{F}$ is 174.88. Thus, the Lipschitz constant of $\mathbf{f}$ can be estimated as $\gamma=175$. Given the matrix A (as in (4)) with the nominal values $a=5, b=$ $7, c=3$, in order to guarantee a positive definite solution for Riccati matrix equation (34), the constant matrices $\Lambda, \mathbf{Q}_{0}, \mathbf{K}, \mathbf{R}=\boldsymbol{\Lambda}, \mathbf{Q}=\gamma^{2} \boldsymbol{\Lambda}^{-1}+\mathbf{Q}_{0}$ are selected in such a way that the conditions of Lemma 4 are satisfied. Thus, $\mathbf{R}=\boldsymbol{\Lambda}=\operatorname{diag}(102.0833,102.0833,102.0833), \mathbf{Q}_{0}=\mathbf{I}$, $\mathbf{K}=\operatorname{diag}(170.30,182.30,172.30), \mathbf{Q}=\operatorname{diag}(301,301,301)$. With these matrices, the solution for Riccati matrix equation (34) is $\mathbf{P}=\operatorname{diag}(1.7002,1.7002,1.7002)$, and according to Theorem 5, the synchronization error e converges exponentially to zero.

The performance of the control law $\mathbf{u}=-\mathbf{K e}$ is verified by simulation. First, the Xian master system (6) with the initial condition $x_{m}(0)=-1, y_{m}(0)=-1$, and $z_{m}(0)=-2$ and the Xian slave system (7) with the initial condition $x_{s}(0)=$ $-4, y_{s}(0)=1$, and $z_{s}(0)=2$ are built on Simulink ${ }^{\circledR}$. Once the error signal $\mathbf{e}$ is obtained, the controller $\mathbf{u}=-\mathbf{K e}$ is applied to the slave system (7). The results of the simulation using the method ode23tb (stiff/TR-BDF2) with relative tolerance=1e6 and absolute tolerance $=1 \mathrm{e}-7$ are presented in Figures 3-6. As can be appreciated in Figures 3, 4, and 5, the states of the slave system (7) do follow the corresponding states of the master system (6) in spite of the difference between the initial conditions. The exponential convergence of the error signal e is shown in Figure 6. For practical purposes, the convergence to zero is attained in less than 0.1 seconds.

Finally, a comparison is accomplished between the proposed technique and active control. As mentioned in Introduction, active control is based on the compensation of the nonlinearities and the decoupling of the synchronization error dynamics. Given the slave system (7) and the master system (6) for Xian system (1), the synchronization error dynamics can be determined as

$$
\dot{e}_{1}=-a e_{1}+x_{s} z_{s}-x_{m} z_{m}+y_{s} z_{s}-y_{m} z_{m}+u_{1}
$$




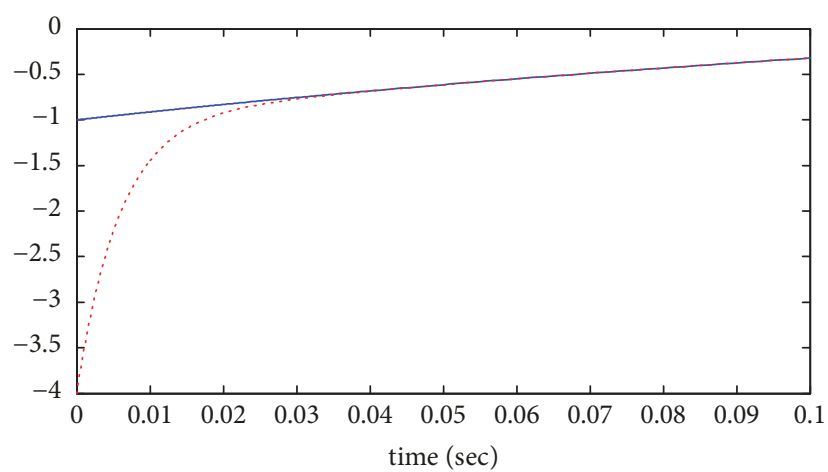

$$
-\mathrm{x}_{\mathrm{m}}
$$$$
\cdots \mathrm{x}_{\mathrm{S}}
$$

FIGURE 3: Synchronization process between slave state $x_{s}$ and master state $x_{m}$.

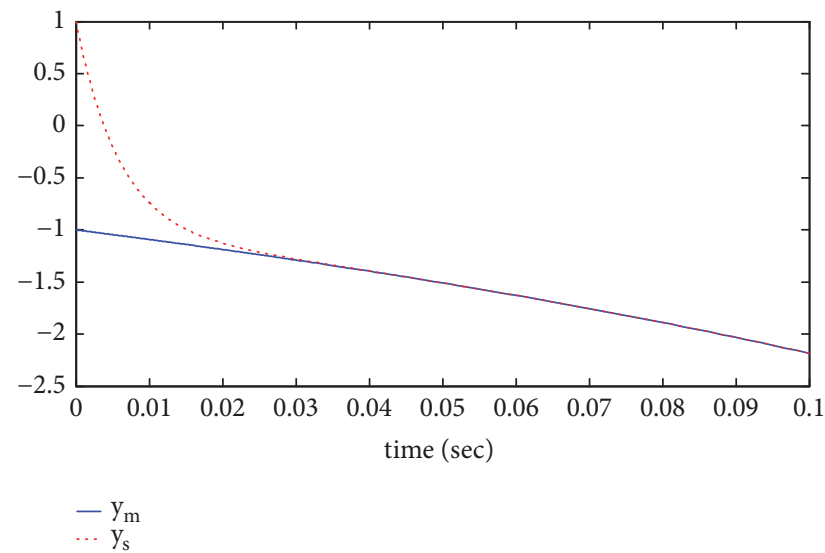

FIGURE 4: Synchronization process between slave state $y_{s}$ and master state $y_{m}$.

$$
\begin{aligned}
& \dot{e}_{2}=b e_{2}-x_{s} z_{s}+x_{m} z_{m}+u_{2} \\
& \dot{e}_{3}=-c e_{3}+x_{s} y_{s}-x_{m} y_{m}+u_{3}
\end{aligned}
$$

However, it should be taken into account that

$$
\begin{aligned}
& x_{s} z_{s}-x_{m} z_{m}=e_{1} e_{3}+z_{m} e_{1}+x_{m} e_{3} \\
& y_{s} z_{s}-y_{m} z_{m}=e_{2} e_{3}+z_{m} e_{2}+y_{m} e_{3} \\
& x_{s} y_{s}-x_{m} y_{m}=e_{1} e_{2}+y_{m} e_{1}+x_{m} e_{2}
\end{aligned}
$$

By substituting (65), (66), and (67) into (64), the synchronization error dynamics can be expressed as

$$
\begin{aligned}
\dot{e}_{1}= & -a e_{1}+e_{1} e_{3}+z_{m} e_{1}+x_{m} e_{3}+e_{2} e_{3}+z_{m} e_{2}+y_{m} e_{3} \\
& +u_{1} \\
\dot{e}_{2}= & b e_{2}-e_{1} e_{3}-z_{m} e_{1}-x_{m} e_{3}+u_{2} \\
\dot{e}_{3}= & -c e_{3}+e_{1} e_{2}+y_{m} e_{1}+x_{m} e_{2}+u_{3}
\end{aligned}
$$

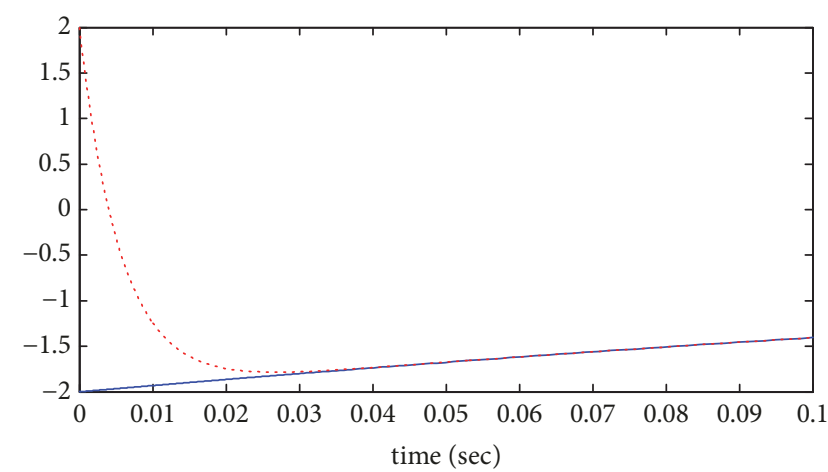

$-\mathrm{Z}_{\mathrm{m}}$
$\cdots \mathrm{Z}_{\mathrm{s}}$

FIGURE 5: Synchronization process between slave state $z_{s}$ and master state $z_{m}$.

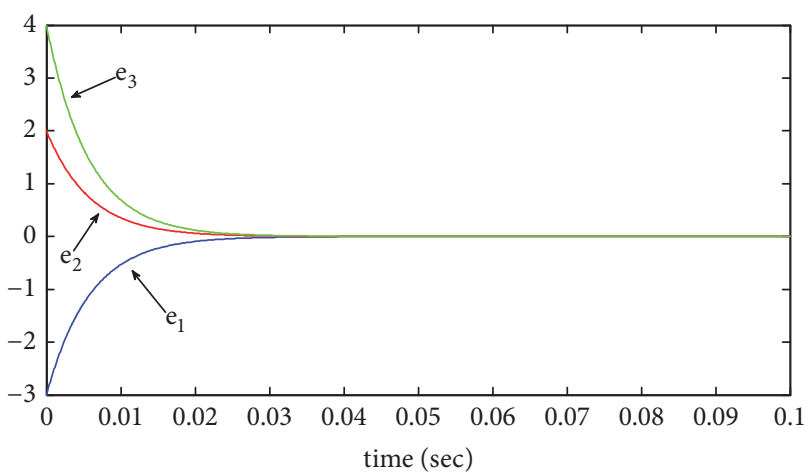

FIGURE 6: Time evolution of synchronization error signals $e_{1}, e_{2}$, and $e_{3}$.

Thus, the corresponding active control is given by

$$
\begin{aligned}
u_{1}= & -e_{1} e_{3}-z_{m} e_{1}-x_{m} e_{3}-e_{2} e_{3}-z_{m} e_{2}-y_{m} e_{3} \\
& -k_{1} e_{1} \\
u_{2}= & e_{1} e_{3}+z_{m} e_{1}+x_{m} e_{3}-k_{2} e_{2} \\
u_{3}= & -e_{1} e_{2}-y_{m} e_{1}-x_{m} e_{2}-k_{3} e_{3}
\end{aligned}
$$

With the objective of accomplishing a systematic comparison between both techniques, the following performance index is used:

$$
J=\int_{0}^{T_{1}}\left(\ell_{1} e_{1}^{2}+\ell_{2} e_{2}^{2}+\ell_{3} e_{3}^{2}+\ell_{4} u_{1}^{2}+\ell_{5} u_{2}^{2}+\ell_{6} u_{3}^{2}\right) d t
$$

where $\ell_{1}, \ldots, \ell_{6}$ are weighting factors and $T_{1}$ is final time. Considering a standard weighting where each term has the same importance and the signals are normalized, the following values should be used: $\ell_{1}=1 / 54, \ell_{2}=1 / 24, \ell_{3}=$ $1 / 96, \ell_{4}=1 / 1,566,726, \ell_{5}=1 / 799,350, \ell_{6}=1 / 2,856,600$, and $T_{1}=0.1 \mathrm{sec}$. To realize a fair comparison, the same values for the gains $k_{1}, k_{2}$, and $k_{3}$ are used in both cases, that is, $k_{1}=170.30, k_{2}=182.30$, and $k_{3}=172.30$. The results are presented in Figures 7 and 8. 


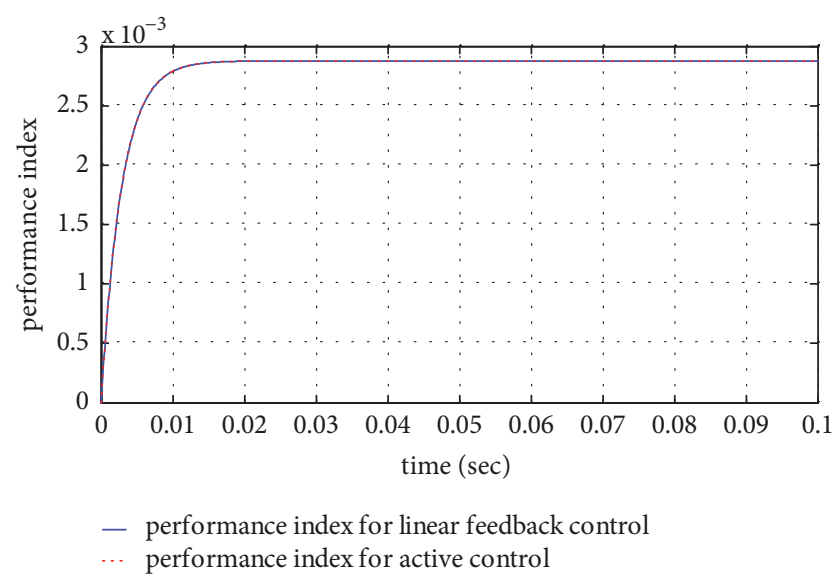

Figure 7: Performance index for linear feedback control and active control.

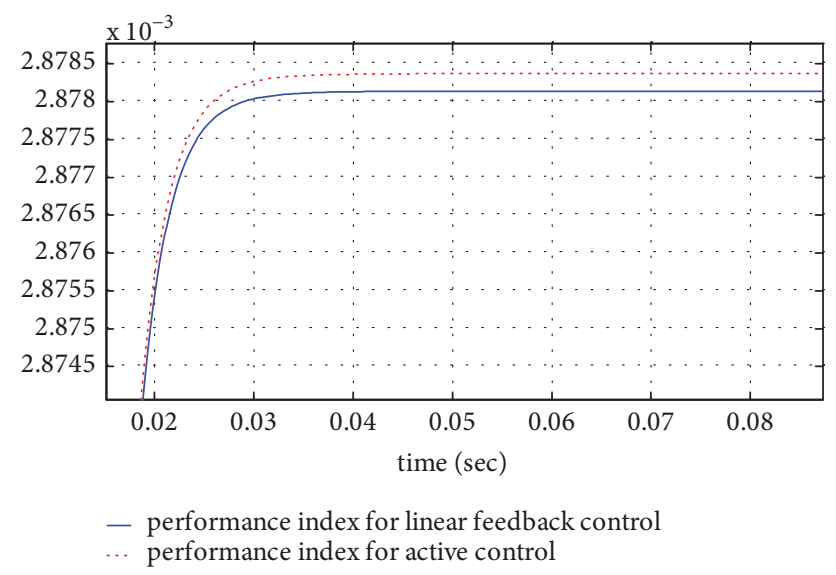

FIgURE 8: Zoom of Figure 7.

As can be appreciated in Figure 7, the performance is almost the same for both cases. In Figure 8, it can be seen that linear feedback control produces slightly lower values for performance index. The apparent similarity in the performance of both techniques is due to the high values used for the gains. However, although the performances were similar in both cases, the implementation of linear feedback control is considerably easier with respect to active control.

\section{Conclusion}

The main attractiveness of the linear feedback is its simple structure being very convenient for practical purposes. In this paper, a linear feedback controller based on an algebraic Riccati equation was presented. To use this controller, first, the Lipschitz constant of nonlinear function of the chaotic system on its attractor must be determined. To achieve this objective, it is necessary to find the maximum values of the states of chaotic system. Although this could be calculated analytically, for simplicity, such values are determined by simulation. Next, values for the gain matrix $\mathbf{K}$ and the matrices of the Riccati equation, $\mathbf{Q}_{0}, \mathbf{Q}$, and $\mathbf{R}$, are selected in such a way that this equation has a unique positive definite solution. Consequently, according to Theorem 5, it is possible to guarantee the exponential convergence to zero of the synchronization error. The strategy is tested on two identical chaotic systems, master and slave, based on Xian system. Numerical simulation confirms the satisfactory performance of the suggested approach. In a future work, a design procedure to obtain systematically the controller gains will be provided.

\section{Data Availability}

No experimental data were used to support this study. The simulation results can be reproduced with Matlab and/or Simulink just using the mathematical model of Xian system and the proposed controller.

\section{Conflicts of Interest}

The authors declare that there are no conflicts of interest regarding the publication of this paper.

\section{Acknowledgments}

This work was financed by COFAA and SIP, Instituto Politécnico Nacional, under Grant 20190052. We acknowledge the support of EDI-IPN and SNI-Conacyt.

\section{References}

[1] T. Hernández Cortés, A. V. Curtidor López, J. Rodríguez Valdez, J. A. Meda Campaña, R. Tapia Herrera, and J. J. Rubio, "Synchronization of discrete-time chaotic fuzzy systems by means of fuzzy output regulation using genetic algorithm," Mathematical Problems in Engineering, vol. 2015, Article ID 198371, 18 pages, 2015.

[2] J. H. Pérez, M. Figueroa, S. A. Rodríguez, and A. López, "Synchronization of chaotic Akgul system by means of feedback linearization and pole placement," IEEE Latin America Transactions, vol. 15, no. 2, pp. 249-256, 2017.

[3] M. Chadli and I. Zelinka, "Chaos synchronization of unknown inputs Takagi-Sugeno fuzzy: application to secure communications," Computers \& Mathematics with Applications, vol. 68, no. 12, pp. 2142-2147, 2014.

[4] C. Aguilar-Ibañez, R. Martinez-Guerra, R. Aguilar-López, and J. L. Mata-Machuca, "Synchronization and parameter estimations of an uncertain Rikitake system," Physics Letters A, vol. 374, no. 35, pp. 3625-3628, 2010.

[5] E. E. Mahmoud and F. S. Abood, "A general formula of complex synchronizations with complex scaling diagonal matrix and time lag," Results in Physics, vol. 12, pp. 603-614, 2019.

[6] E. E. Mahmoud and F. S. Abood, "A novel sort of adaptive complex synchronizations of two indistinguishable chaotic complex nonlinear models with uncertain parameters and its applications in secure communications," Results in Physics, vol. 7, pp. 4174-4182, 2017.

[7] R. Luo, H. Su, and Y. Zeng, "Chaos control and synchronization via switched output control strategy," Complexity, vol. 2017, Article ID 6125102, 11 pages, 2017. 
[8] R. Luo and H. Su, "The robust control and synchronization of a class of fractional-order chaotic systems with external disturbances via a single output," Complexity, vol. 2018, Article ID 1984348, 8 pages, 2018.

[9] Q. Ye, Z. Jiang, and T. Chen, "Adaptive feedback control for synchronization of chaotic neural systems with parameter mismatches," Complexity, vol. 2018, Article ID 5431987, 8 pages, 2018.

[10] T. Youssef, M. Chadli, H. R. Karimi, and M. Zelmat, "Chaos synchronization based on unknown input proportional multipleintegral fuzzy observer," Abstract and Applied Analysis, vol. 2013, Article ID 670878, 11 pages, 2013.

[11] M. Chadli, I. Zelinka, and T. Youssef, "Unknown inputs observer design for fuzzy systems with application to chaotic system reconstruction," Computers \& Mathematics with Applications, vol. 66, no. 2, pp. 147-154, 2013.

[12] E. E. Mahmoud and M. A. Al-Adwani, "Dynamical behaviors, control and synchronization of a new chaotic model with complex variables and cubic nonlinear terms," Results in Physics, vol. 7, pp. 1346-1356, 2017.

[13] J. A. Meda-Campaña, B. Castillo-Toledo, and G. Chen, "Synchronization of chaotic systems from a fuzzy regulation approach," Fuzzy Sets and Systems, vol. 160, no. 19, pp. 28602875, 2009.

[14] V. R. Nosov, H. Dominguez, J. A. Ortega-Herrera, and J. A. Meda-Campaña, "Complex dynamics and chaos in commutable pendulum," Revista Mexicana de Fisica, vol. 58, pp. 6$12,2012$.

[15] A. Khan, D. Khattar, and N. Prajapati, "Dual combination combination multi switching synchronization of eight chaotic systems," Chinese Journal of Physics, vol. 55, no. 4, pp. 1209-1218, 2017.

[16] P. Gholamin and A. R. Sheikhani, "A new three-dimensional chaotic system: Dynamical properties and simulation," Chinese Journal of Physics, vol. 55, no. 4, pp. 1300-1309, 2017.

[17] A. Khan, M. Budhraja, and A. Ibraheem, "Multi-switching dual compound synchronization of chaotic systems," Chinese Journal of Physics, vol. 56, no. 1, pp. 171-179, 2018.

[18] I. Zelinka, M. Chadli, D. Davendra, R. Senkerik, and R. Jasek, "An investigation on evolutionary reconstruction of continuous chaotic systems," Mathematical and Computer Modelling, vol. 57, no. 1-2, pp. 2-15, 2013.

[19] P. P. Singh, J. P. Singh, and B. K. Roy, "Synchronization and antisynchronization of Lu and Bhalekar-Gejji chaotic systems using nonlinear active control," Chaos, Solitons \& Fractals, vol. 69, pp. 31-39, 2014.

[20] P. Singh and B. Roy, "Comparative performances of synchronisation between different classes of chaotic systems using three control techniques," Annual Reviews in Control, vol. 45, pp. 152165, 2018.

[21] S. Çiçek, A. Ferikoğlub, and I. Pehlivan, "A new 3D chaotic system: dynamical analysis, electronic circuit design, active control synchronization and chaotic masking communication application," Optik - International Journal for Light and Electron Optics, vol. 127, no. 8, pp. 4024-4030, 2016.

[22] M. Varan and A. Akgul, "Control and synchronisation of a novel seven-dimensional hyperchaotic system with active control," Pramana-Journal of Physics, vol. 90, no. 4, 2018.

[23] M. Varan, A. Akgül, E. Güleryüz, and K. Serbest, "Synchronisation and circuit realisation of chaotic hartley system," Zeitschrift fur Naturforschung - Section A Journal of Physical Sciences, vol. 73, no. 6, pp. 521-531, 2018.
[24] M. T. Yassen, "Chaos synchronization between two different chaotic systems using active control," Chaos, Solitons \& Fractals, vol. 23, no. 1, pp. 131-140, 2005.

[25] U. Vincent and J. Laoye, "Synchronization and control of directed transport in chaotic ratchets via active control," Physics Letters A, vol. 363, no. 1-2, pp. 91-95, 2007.

[26] A. Uçar, K. E. Lonngren, and E. W. Bai, "Synchronization of the unified chaotic systems via active control," Chaos, Solitons and Fractals, vol. 27, pp. 1292-1297, 2006.

[27] U. E. Vincent, "Synchronization of Rikitake chaotic attractor using active control," Physics Letters A, vol. 343, no. 1-3, pp. 133138, 2005.

[28] Y. M. Lei, W. Xu, and H. C. Zheng, "Synchronization of two chaotic nonlinear gyros using active control," Physics Letters A, vol. 343, no. 1-3, pp. 153-158, 2005.

[29] Y. Lei, W. Xu, and W. Xie, "Synchronization of two chaotic fourdimensional systems using active control," Chaos, Solitons \& Fractals, vol. 32, no. 5, pp. 1823-1829, 2007.

[30] U. E. Vincent, "Synchronization of identical and non-identical 4-D chaotic systems using active control," Chaos, Solitons \& Fractals, vol. 37, no. 4, pp. 1065-1075, 2008.

[31] V. Sundarapandian and R. Karthikeyan, "Hybrid synchronization of hyperchaotic lorenz and hyperchaotic chen systems via active control," Journal of Engineering and Applied Sciences, vol. 7, no. 3, pp. 254-264, 2012.

[32] J. H. Pérez-Cruz, D. Zenteno-Lara, D. Ávila-González, and C. Nwachioma, "Synchronization of multi-character nazarimehr system using active control," International Journal of Scientific and Engineering Research, vol. 9, pp. 438-444, 2018.

[33] M. Chen and Z. Han, "Controlling and synchronizing chaotic Genesio system via nonlinear feedback control," Chaos, Solitons \& Fractals, vol. 17, no. 4, pp. 709-716, 2003.

[34] L. Huang, R. Feng, and M. Wang, "Synchronization of chaotic systems via nonlinear control," Physics Letters A, vol. 320, no. 4, pp. 271-275, 2004.

[35] J. H. Park, "On synchronization of unified chaotic systems via nonlinear control," Chaos, Solitons and Fractals, vol. 25, pp. 699704, 2005.

[36] H.-K. Chen, "Global chaos synchronization of new chaotic systems via nonlinear control," Chaos, Solitons \& Fractals, vol. 23, no. 4, pp. 1245-1251, 2005.

[37] Q. Zhang and J. Lu, "Chaos synchronization of a new chaotic system via nonlinear control," Chaos, Solitons and Fractals, vol. 37, pp. 175-179, 2008.

[38] M. Mossa Al-Sawalha and M. S. M. Noorani, "Antisynchronization of two hyperchaotic systems via nonlinear control," Communications in Nonlinear Science and Numerical Simulation, vol. 14, no. 8, pp. 3402-3411, 2009.

[39] M. Mossa Al-sawalha and M. S. M. Noorani, "On antisynchronization of chaotic systems via nonlinear control," Chaos, Solitons \& Fractals, vol. 42, no. 1, pp. 170-179, 2009.

[40] S. Zheng, "Multi-switching combination synchronization of three different chaotic systems via nonlinear control," Optik International Journal for Light and Electron Optics, vol. 127, no. 21, pp. 10247-10258, 2016.

[41] J. H. Pérez-Cruz, E. A. Portilla-Flores, P. A. Niño-Suárez, and R. Rivera-Blas, "Design of a nonlinear controller and its intelligent optimization for exponential synchronization of a new chaotic system," Optik - International Journal for Light and Electron Optics, vol. 130, pp. 201-212, 2017. 
[42] X. Wu, G. Chen, and J. Cai, "Chaos synchronization of the master-slave generalized Lorenz systems via linear state error feedback control," Physica D: Nonlinear Phenomena, vol. 229, no. 1, pp. 52-80, 2007.

[43] M. Rafikov and J. M. Balthazar, "On control and synchronization in chaotic and hyperchaotic systems via linear feedback control," Communications in Nonlinear Science and Numerical Simulation, vol. 13, no. 7, pp. 1246-1255, 2008.

[44] Y. Chen, X. Wu, and Z. Gui, "Global synchronization criteria for a class of third-order non-autonomous chaotic systems via linear state error feedback control," Applied Mathematical Modelling: Simulation and Computation for Engineering and Environmental Systems, vol. 34, no. 12, pp. 4161-4170, 2010.

[45] J. J. Rubio, "Robust feedback linearization for nonlinear processes control," ISA Transactions ${ }^{\circledR}$, vol. 74, pp. 155-164, 2018.

[46] Y. Wang, Z. Guan, and H. O. Wang, "Feedback and adaptive control for the synchronization of Chen system via a single variable," Physics Letters A, vol. 312, no. 1-2, pp. 34-40, 2003.

[47] J. Lu, X. Wu, X. Han, and J. Lü, "Adaptive feedback synchronization of a unified chaotic system," Physics Letters A, vol. 329, no. 4-5, pp. 327-333, 2004.

[48] X. P. Han, J.-A. Lu, and X. Q. Wu, "Adaptive feedback synchronization of Lü system," Chaos, Solitons \& Fractals, vol. 22, no. 1, pp. 221-227, 2004.

[49] M. T. Yassen, "Controlling chaos and synchronization for new chaotic system using linear feedback control," Chaos, Solitons \& Fractals, vol. 26, no. 3, pp. 913-920, 2005.

[50] H. Chen, "Chaos control and global synchronization of Liu chaotic systems using linear balanced feedback control," Chaos, Solitons \& Fractals, vol. 40, no. 1, pp. 466-473, 2009.

[51] X. Wang and Y. Wang, "Adaptive control for synchronization of a four-dimensional chaotic system via a single variable," Nonlinear Dynamics, vol. 65, no. 3, pp. 311-316, 2011.

[52] C. C. Yang, "Adaptive synchronization of Lü hyperchaotic system with uncertain parameters based on single-input controller," Nonlinear Dynamics, vol. 63, no. 3, pp. 447-454, 2011.

[53] Y. Xian, C. Xia, T. Guo, K. Fu, and C. Xu, "Dynamical analysis and FPGA implementation of a large range chaotic system with coexisting attractors," Results in Physics, vol. 11, pp. 368-376, 2018.

[54] Q. Lai, A. Akgul, M. Varan, J. Kengne, and A. Turan Erguzel, "Dynamic analysis and synchronization control of an unusual chaotic system with exponential term and coexisting attractors," Chinese Journal of Physics, vol. 56, pp. 2837-2851, 2018.

[55] S. Raghavan and J. K. Hedrick, "Observer design for a class of nonlinear systems," International Journal of Control, vol. 59, no. 2, pp. 515-528, 1994.

[56] H. K. Khalil, Nonlinear Systems, Pearson, 3rd edition, 2002.

[57] H. Márquez, Nonlinear Control Systems: Analysis and Design, Wiley-Interscience, New Jersey, NJ, USA, 1st edition, 2003.

[58] A. Poznyak, E. N. Sanchez, and W. Yu, Differential Neural Networks for Robust Nonlinear Control-Identification, State Estimation, and Trajectory Tracking, World Scientific Publishing Company, 1st edition, 2001.

[59] J. H. Pérez-Cruz, J. J. Rubio, E. Ruiz-Velázquez, and G. SolísPerales, "Tracking control based on recurrent neural networks for nonlinear systems with multiple inputs and unknown deadzone," Abstract and Applied Analysis, vol. 2012, Article ID 471281, 18 pages, 2012.

[60] J. H. Pérez-Cruz, J. D. J. Rubio, R. Encinas, and R. Balcazar, "Singularity-free neural control for the exponential trajectory tracking in multiple-input uncertain systems with unknown deadzone nonlinearities," The Scientific World Journal, vol. 2014, 10 pages, 2014. 


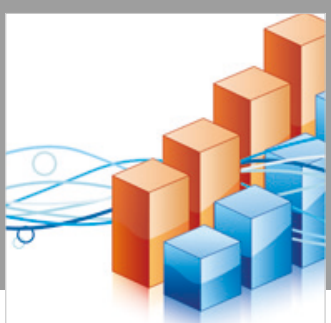

Advances in

Operations Research

\section{-n-m}
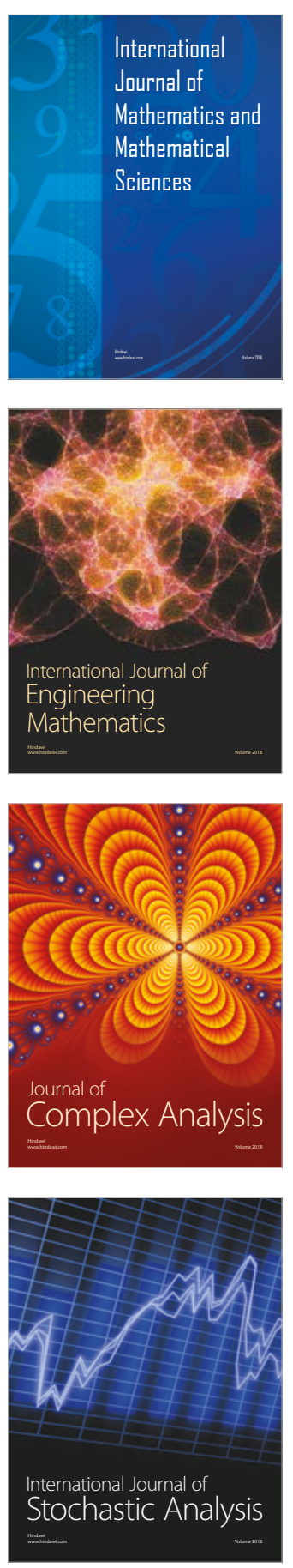
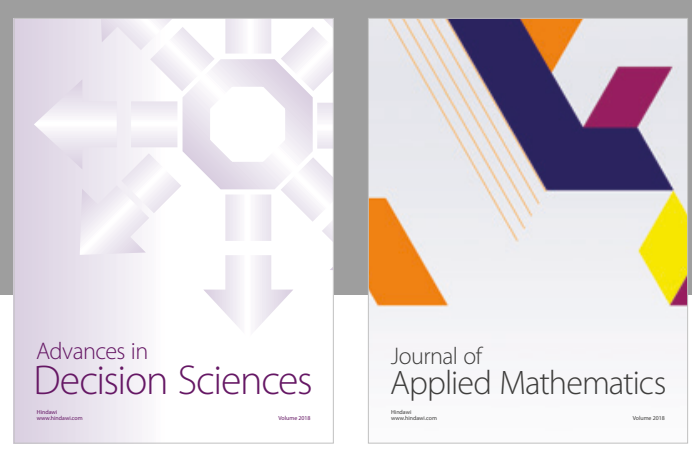

Journal of

Applied Mathematics
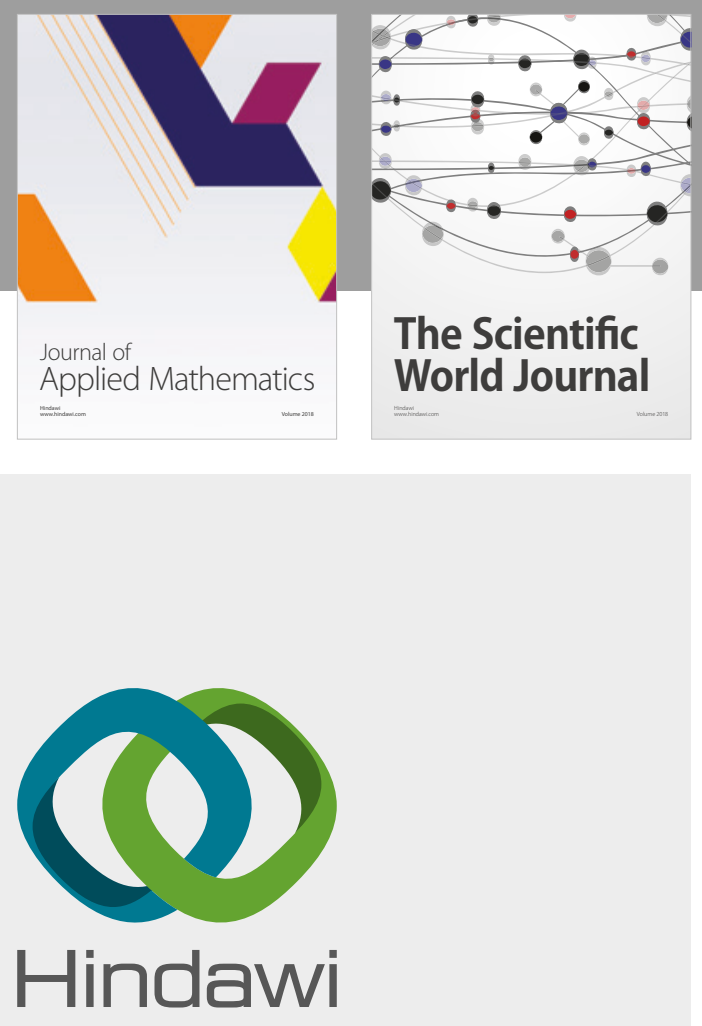

Submit your manuscripts at

www.hindawi.com

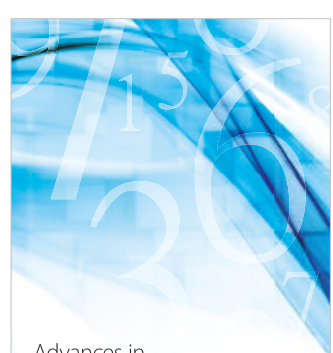

Advances in
Numerical Analysis
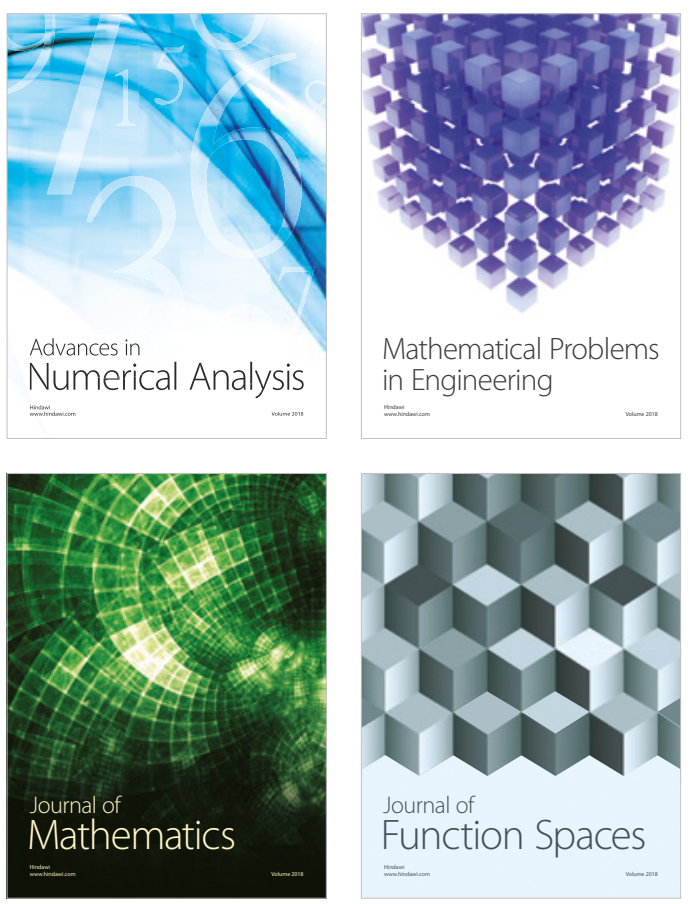

Mathematical Problems in Engineering

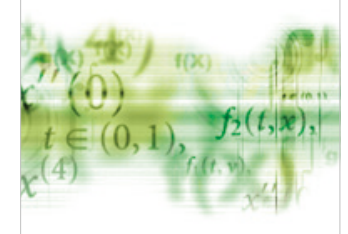

International Journal of

Differential Equations

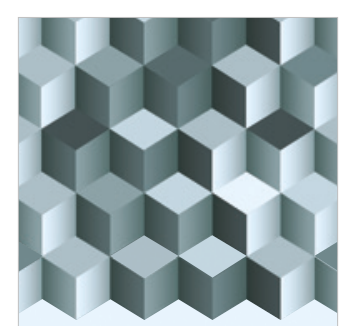

Journal of

Function Spaces
The Scientific

World Journal

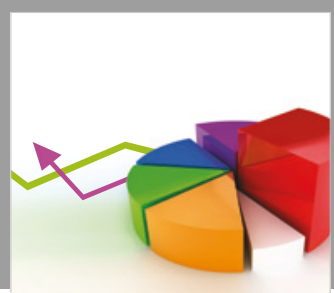

Journal of

Probability and Statistics
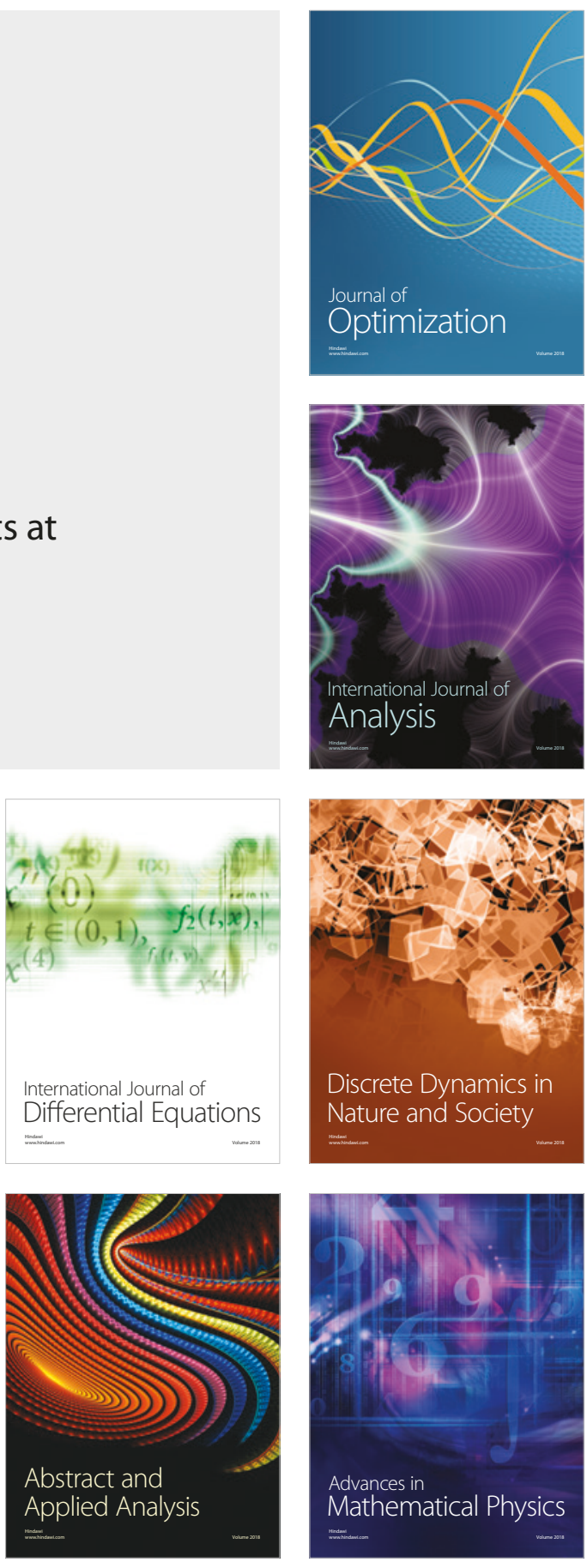